\title{
VALIDEZ ATHÉTICA
}

\section{El paradigma: validez thética vs. validez athética}

\subsection{Los términos del paradigma}

1 paradigma: validez thética vs. validez athética ha sido formulado en una reciente contribución a la deóntica de la validez: Amedeo G. Conte, Minima deontica, 1988.

1.1.1. La validez thética (en italiano: validità thética, en alemán: thetische Gültigkeit, en inglés: thetic validity, en francés: validité thétique) es la validez deóntica (validità deontica, deontische Gultigkeit, deontic validity, validité deontique) que es el producto (el wytwór, en el léxico del filósofo polaco Kazimierz Twardowski) de un acto thético de posición, de un acto de thésis.

El adjetivo «thético» alude al sustantivo griego «thésis», que equivale al español «posición», al italiano «posizione», al alemán «Setzung».

1.1.2. La validez athética (validità athética, athetische Gültigkeit, athetic validity, validité athétique) es la validez no thética, o sea la validez deóntica que no es el producto (el wytwór) de un acto thético de posición, de un acto de thésis.

El término «athético» se forma, manifiestamente, prefijando «thético» con un alpha privativum (cfr. «abúlico», «anómico», «arrítmico»).

\subsection{El estatuto del paradigma}

1.2.1. La elaboración del concepto de validez athética es una tarea puramente teorética, sin presuposiciones ni implicaciones paralelas a la teoría filosófica. La elaboración del concepto (Begriff) de 
validez athética no es el reflejo de (y no se refleja en) ninguna concepción (Auffassung) de la validez deóntica.

1.2.1.1. Si existen presuposiciones e implicaciones ateoréticas no es por el reconocimiento del fenómeno de la validez athética sino, al contrario, por su desconocimiento. El desconocimiento de la validez athética, o sea la reducción acrítica de la validez deóntica a validez thética (la contracción de la validez deóntica en validez thética), es el punto de partida de la concepción de la validez deóntica que es propia del positivismo jurídico.

1.2.1.2. Que el concepto (Begriff) de validez athética no esté ligado a una determinada concepción (Auffassung) de la validez deóntica lo prueba el hecho de que el fenómeno de la validez athética está documentado en dos filósofos del derecho con Weltanschauungen opuestas y estilos filosóficos opuestos: Giovanni Tarello (Teorie e ideologie nel diritto sindacale, 1967) y Luigi Lombardi Vallauri (Saggio sul diritto giurisprudenziale, 1967).

Tanto al derecho sindical estudiado por Tarello como al derecho jurisprudencial paralelamente (y sincrónicamente) estudiado por Lombardi Vallauri le es propia una validez deóntica que no es thética: una validez deóntica athética.

\subsection{Fecundidad heurística del paradigma}

El paradigma: validez thética vs. validez athética es heurísticamente fecundo tanto en la teoría de la validez deóntica como en la metateoría de las teorías de la validez deóntica.

1.3.1. En primer lugar, el paradigma: validez thética vs. validez athética es heurísticamente fecundo en la teoría de la validez deóntica.

A la luz del mismo es posible reconocer, y conocer, fenómenos deónticos anómalos, formas de validez deóntica frecuentemente desconocidas precisamente por su anomalía, por su atipicidad.

1.3.2. En segundo lugar, el paradigma: validez thética vs. validez athética es heurísticamente fecundo en la metateoría de las teorías de la validez deóntica.

En particular, a la luz del mismo es posible detectar y superar una paradoja que parece estar presente en la contribución que un eminente filósofo de las ciencias sociales, Theodor Geiger, ha apor- 
lado a la deóntica de la validez en su libro: Vorstudien zu einer Soziologie des Rechts, Estudios de sociología del derecho, $1947^{1}$.

\section{Una paradoja}

\subsection{Paradigmas de la paradoja}

Las Vorstudien zu einer Soziologie des Rechts, Estudios de sociología del derecho, 1947, de Theodor Geiger no son una obra de deóntica de la validez.

Pero en las Vorstudien aparecen tres paradigmas que son relevantes para la deóntica y para la metadeóntica.

Los tres paradigmas son:

(i) regularidad deóntica vs. regularidad adeóntica;

(ii) norma vs. enunciado deóntico;

(iii) enunciado deóntico proclamativo vs. enunciado deóntico declarativo.

${ }^{1}$ En el presente ensayo el adjetivo «thético» («thético», «thetisch», «thetic», «thétique») se predica de la validez deóntica (validità deontica, deontische Gültigkeit, deontic validity, validité déontique) y no de normas (en ninguno de los sentidos del término «norma»).

En cambio, «thético» aparece frecuentemente como predicado de normas en obras de autores polacos, en las que (a partir de Czeslaw Znamierowski) suele aparecer el sintagma «norma tetyczna», «thetische Norm», "thetic norm», «norma thetica», "norma thética». En A. G. Conte (Aspekte der Semantik der deontischen Sprache, 1977; Aspetti della semantica del linguaggio deontico, 1977; Parerga leibnitiana, 1978; Nove studi sul linguaggio normativo, 1985; Mínima deontica, 1988) se enumeran obras de autores polacos (en polaco: Jerzy Kalinowski, Kazimierz Opalek, Zygmunt Ziembinski; en alemán: Kazimierz Opalek, Ryszard Sarkowicz; en italiano: Feliks Bednarski) en las que aparece «tetyczny» o su correlativo en otros idiomas.

Inexplicablemente, algunos estudiosos polacos emplean el adjetivo polaco «telyczny» sin tener en cuenta su etimología ("thésis», «thetikós»).

Una prueba de su confusión sobre el origen etimológico de este adjetivo es el modo en que estos estudiosos traducen «tetyczny» a idiomas en los que (a diferencia de lo que ocurre en polaco) la theta griega se reproduce mediante el digrama «th». Junto a las grafías correctas «thetic» y «thetisch», he encontrado tanto «tethic» (en la que se invierten «t» $\mathrm{y}$ «th») como «tetisch» (en la que se sustituye el digrama «th» por «t»).

G. P. M. Azzoni (quien ha contribuido originalmente a la elaboración del paradigma: validez thética vs. validez athética) señala el sugerente paralelismo entre este paradigma y el paradigma de Friedrich August von Hayek: thesis vs. nomos. La relevancia filosófica de Hayek ha sido puesta de manifiesto por Enrico di Robilant y Mauro Barberis. 
Haré explícitos estos tres paradigmas, valiéndome libremente de mis conceptos y de mi léxico, en los epígrafes 2.1.1., 2.1.2., 2.1.3., del presente ensayo Validez athética.

2.1.1. Primer paradigma: regularidad deóntica vs. regularidad adeóntica. regularidad:

2.1.1.1. Los términos del primer paradigma de Geiger son dos especies de

(i) regularidad deóntica («Regelmässigkeit»);

(ii) regularidad adeóntica («Regelhaftigkeit»).

2.1.1.2. La distinción entre regularidad deóntica y regularidad adeóntica es explicada por Alberto Febbrajo en Materiali sulla sociologia del diritto di Theodor Geiger, 1979. Un punto de referencia de Febbrajo es el ensayo Codici deontici, 1976, de Amedeo G. Conte.

2.1.1.2.1. Esta es la cuestión planteada en Codici deontici:

¿Existe un discrimen entre el (deónticamente no neutro) seguir una regla y el (deónticamente neutro) proseguir en una regularidad?

2.1.1.2.2. Y ésta es la respuesta dada en Codici deontici:

Existe un discrimen entre el (deónticamente no neutro) seguir una regla y el (deónticamente neutro) proseguir en una regularidad. Tal discrimen es inherente a lo que Ludwig Wingenstein llamara la Grammatik de los términos «regla» y «regularidad».

2.1.1.2.2.1. Una regularidad es (por definición) simple, una, necesariamente idéntica a sí misma. y bina.

2.1.1.2.2.2. No ocurre lo mismo con una regla; de hecho, una regla puede ser una deontici).

Una regla es una y bina en la paradoja proerética de Orestes (estudiada en Codici

La regla que ordena a Orestes honrar a sus padres es violada actúe como actúe Orestes.

La viola si venga a su padre y la viola si no venga a su padre. «La regla deónticamente una es, en este caso, proeréticamente bina» ${ }^{2}$.

${ }^{2}$ A la tesis formulada por A. G. Conte, Codici deontici, 1976, sobre las relaciones entre reglas y regularidades (toda regularidad es necesariamente una; una regla 
2.1.1.2.3. De la cuestión conceptual de si existe un discrimen entre proseguir en una regularidad y seguir una regla (cuestión discutida en Amedeo G. Conte, Codici deontici, 1976) se distingue la cuestión epistemológica de si es posible inferir (por inducción, o, como pienso, por abducción: abduction es un concepto de Charles Sanders Pierce), de la observación de la acción, qué regla sigue la acción.

2.1.1.2.3.1. He analizado este tema en referencia a las reglas eidético-constitutivas. A modo de complemento de lo que he escrito en otro lugar, resaltaré ahora brevemente las presuposiciones (presupposizioni, Präsuppositionen, presuppositions, présuppositions) de la cuestión de qué regla sigue la acción observada.

La cuestión de qué regla sigue la acción tiene dos presuposiciones:

(i) Primera presuposición: presuposición de no anomicidad (de ananomicidad).

La primera presuposición es que la acción no es anómica (la acción no es anómica si, y sólo si, el agente sigue al menos una regla).

(ii) Segunda presuposición: presuposición de no idionomicidad (de anidionomicidad).

puede ser una y bina) es sólo externamente afín la tesis formulada por R. Brown, Rules and Laws in Sociology, 1973, págs. 98, sobre las relaciones entre reglas (《rules») y leyes («laws»): «Two existing rules can be incompatible, whereas two laws cannot both hold».

La tesis de Robert Brown parece una verdad de razón (una "vérité de raison»); pero es una falsedad de hecho. En realidad, es falso que todas las reglas sean susceptibles de contradicción (sean posibles objetos de antinomia): existen reglas (las reglas eidético-constitutivas y las reglas noético-constitutivas) respecto a las cuales la contradicción (la antinomia) no es posible. De hecho, como ha demostrado A. G. Conte, las reglas eidético-constitutivas (y las reglas noético-constitutivas) sí son «rules», pero, igual que las «laws» de Robert Brown, no son susceptibles de contradicción (no pueden ser antinómicas).

La imposibilidad de antinomia (imposibilidad que concierne a las reglas eidético-constitutivas y a las reglas noético-constitutivas) no concierne, genéricamente, a todas las especies de reglas constitutivas (constitutivas son las reglas que o son condición de aquello de lo que son reglas, o ponen una condición de aquello de lo que son regla). Por el contrario: respecto a las otras cuatro especies de reglas constitutivas (reglas thético-constitutivas, reglas anancástico-constitutivas, reglas metatético-constitutivas, reglas nómico-constitutivas) es posible la antinomia. Sobre las condiciones de antinomia entre reglas constitutivas ha escrito G. P. M. Azzoni, Il concetto di condizione nella tipologia delle regole, 1988. 
La segunda presuposición es que la acción no es idionómica (la acción no es idionómica si, y sólo si, el agente sigue como máximo una regla; si, y sólo si, ninguno de los comportamientos en los que se articula la acción sigue una propia regla, una regla propia del comportamiento).

2.1.1.2.3.2. Estas son dos presuposiciones de la cuestión de qué regla sigue la acción.

Ahora bien, de la mera observación de la acción no se infiere ni que se satisface la primera presuposición ni que se satisface la segunda. De ninguna de las dos presuposiciones se puede determinar, sobre la base de la mera observación de la acción, si se satisface. En otras palabras: de la mera observación de la acción no se infiere ni que la acción no es anómica (es ananómica) ni que la acción no es idionómica (es anidionómica) ${ }^{3}$.

2.1.1.3. Remitiéndose originalmente a Codici deontici, Alberto Febbrajo, Materiali sulla sociologia del diritto di Theodor Geiger, 1979, interpreta así la distinción (efectuada por Geiger) entre regularidad adeóntica y regularidad deóntica, entre «faktische Regelhaftikeit» $\mathrm{y}$ «sozial geforderte Regelmassigkeit»:

(i) la adeóntica Regelhaftigkeit es proseguir una regularidad;

(ii) la deóntica Regelmassigkeit es seguir una regla ${ }^{4}$.

${ }^{3}$ Un breve comentario sobre el léxico que empleo. El adjetivo «idionómico» y el sustantivo «idionomía» son neologismos míos. Su etimología es clara: el sustantivo griego «nomos» (que corresponde al italiano «regola») y el adjetivo griego «idios» (que equivale al italiano «proprio», al alemán «eigen»). Y, ostensiblemente, son neologismos míos «ananómico» y «ananomía», por una parte, $\mathrm{y}$ «anidionómico» $\mathrm{y}$ «anidionomia», por otra (cuatro términos formados al añadir como prefijo un alpha privativum).

${ }^{4}$ La distinción de Geiger entre regularidad adeóntica y regularidad deóntica es una distinción entre dos especies de regularidades. De esta distinción se distingue otra (formulada por F. A. Von Hayek, Law, Legislation and Liberty, 1982, vol. I, págs. 78-79) que no es una distinción entre dos especies de regularidades, sino entre dos especies de reglas:

(i) las adeónticas "descriptive rules which assert the regular recurrence of certain sequences of events (including human actions)»;

(ii) las deónticas «normative rules which state that such sequences "ough" to take place». reglas.

Deónticas o adeónticas, respectivamente, son, en Geiger, las regularidades; en Hayek, las

En otras palabras: «deóntico» y «adeóntico» se predican, en Geiger, de regularidades; por el contrario, en Hayek, deonticidad y adeonticidad no se predican de regularidades, sino de reglas («rules»). 
2.1.2. Segundo paradigma: norma vs. enunciado deóntico.

Los términos del segundo de los tres paradigmas de Geiger son dos especies de entidades deónticas:

(i) norma («Norm»);

(ii) enunciado deóntico («Normsatz»).

La tesis de Geiger sobre la relación entre Norm y Normsatz se articula mediante dos subtesis.

La primera subtesis concierne a las relaciones entre la intensión (intensione, Intension, intension, intension) del término «Norm» y la intensión del término «Normsatz».

La segunda subtesis concierne a las relaciones entre la extensión (estensione, Extension, extension, extension) del término «Norm» y la extensión del término «Normsatz».

Ambas subtesis son relevantes para la deóntica y para la metadeóntica.

\subsubsection{Primera subtesis: no sinonimia de «Norm»y «Normsatz».}

Los términos «Norm» $\mathrm{y}$ «Normsatz» no tienen la misma intensión.

Geiger niega que el término «Norm» y el término «Normsatz» sean sinónimos.

2.1.2.2. Segunda subtesis: no correspondencia biunívoca entre el conjunto de las Normen y el conjunto de los Normsätze.

El término «Norm» y el término «Normsatz» no tienen la misma extensión.

Geiger niega que entre el conjunto de las Normen y el conjunto de los Normsätze exista correspondencia biunívoca. En particular: la presencia del enunciado deóntico («Normsatz») no es, para Geiger, ni condición necesaria ni condición suficiente de existencia de la Norm.

2.1.2.2.1. En primer lugar, la presencia de un Normsatz no es, para Geiger, condición necesaria (condizione necessaria, notwendige Bedingung, necessary condition, condition necessaire) de existencia de una Norm.

Geiger afirma explícitamente que «la norma misma [die Norm selbst] puede subsistir incluso sin la envoltura lingüística [sprachliche Hülle] del enunciado»: «die Norm selbst auch olme die sprachliche Hülle des Satzes bestehen kann». 
2.1.2.2.2. En segundo lugar, la presencia de un Normsatz no es, para Geiger, condición suficiente (condizione sufficiente, hinreichende Bedingung, sufficient condition, condition suffisante) de existencia de una Norm.

Geiger afirma explícitamente que «no a todo enunciado con forma gramatical de enunciado deóntico le corresponde una norma subsistente»: «nicht jeder Aussage von der grammatischen Form des Normsatzes eine subsistente Norm entspricht».

En otras palabras: «No todo enunciado que se presente bajo la apariencia de norma verbal contiene [...]: una norma»: «Nicht jeder Satz von der ausseren Gestalt der Wortnorm enthält $[. .$.$] eine Norm».$

2.1.3. Tercer paradigma: enunciado deóntico proclamativo vs. enunciado deóntico declarativo.

Los términos del tercer y último de los tres paradigmas de Geiger son dos especies de enunciados deónticos nomotéticos, dos especies de Normsätze:

(i) enunciados deónticos proclamativos («proklamative Normsätze»);

(ii) enunciados deónticos declarativos («deklarative Normätze»).

\subsubsection{Enunciado deóntico proclamativo.}

El enunciado deóntico proclamativo («proklamativer Normsatz») es el Normsatz a través del cual una Norm es théticamente estatuida («statuiert»), introducida («eingeführt»), creada ("geschafft»).

\subsubsection{Enunciado deóntico declarativo.}

El enunciado deóntico declarativo («deklarativer Normsatz») es, por el contrario, el Normsatz que constata ("Konstatiert», "stellt fest»: Geiger recurre tanto al verbo germánico «feststellen» como al galicismo «konstatieren») la subsistencia («das Bestehen») de una «norma subsistente», y, de tal «subsistente Norm», representa una codificación nomotética 5 .

${ }^{5}$ Tanto «feststellen » como «konstatieren» sonverbos factivos (verbi fattivi, faktive Verben, factive verbs, verbes factifs); es decir, son verbos en los que se presupone (präsupponiert) la verdad de la proposición sobre la que actúan.

Sobre la factivity, cfr. P. Kjparsky/C. Kiparsky, Fact, 1970.

Sobre las relaciones entre factividad y theticidad, A. G. Conte, Aspekte der Semantik der 
2.1.3.2.1. Geiger formula reiteradamente el hermafroditismo deóntico de los deklarative Normsätze.

2.1.3.2.1.1. Un enunciado deóntico declarativo constata la subsistencia de una norma subsistente («konstatiert das Bestehen einer subsistenten Norm») y convalida un modelo de comportamiento (un «eingespieltes Gebarensmodell»).

2.1.3.2.1.2. De la norma subsistente el enunciado deóntico declarativo («deklarativer Normsatz») es expresión y consolidación. En él la norma subsistente se expresa y consolida ("ausgedrückt und verfestigt»), es (como icásticamente escribe Geiger) capturada (eingefangen: «eine [...] subsistente Norm in einem deklarativen Normsatz eingefangen wird» $)^{6}$.

2.1.3.2.2. Resulta filosóficamente provocador que Geiger no sólo advierta la alteridad de la Norm respecto al Normsatz y la no correlatividad entre Normen y Normsatze, sino que, a continuación, afirme que la norma subsistente es la norma en sentido propio («Norm in eigentilichen Sinn») 7 .

2.2. Formulación de la paradoja

2.2.1. Deklarativer Normsatz, enunciado deóntico declarativo: este concepto (cardinal en la obra de Geiger) puede parecer un concepto paradójico, autocontradictorio (así como, simétricamente, puede parecer un concepto tautológico el concepto opuesto de enunciado deóntico proclamativo, proklamativer Normsatz).

2.2.2. De hecho, en el concepto de deklarativer Normsatz parecen coincidir dos rasgos (dos notae, dos Merkmale) incompatibles: el rasgo de la theticidad y el rasgo de la rheticidad ${ }^{8}$.

deontischen Sprache, 1977; A. G. Conte, Aspetti della semantica del linguaggio deontico, 1977.

${ }^{6}$ Un breve comentario sobre el verbo, usado por Geiger, «verfestigen», que corresponde al italiano «consolidare». Como me indican tanto Giampaolo M. Azzoni como Paolo di Lucia, un sustantivo italiano que deriva de "consolidare», concretamente «consolidazione», es un terminus technicus de los historiadores del derecho. Cfr., por ejemplo, M. E. Viora, Consolidazioni e codificazioni, 1932, 1967 (3.aed.).

${ }^{7}$ Cfr. T. Geiger, Vorstudien zu einer Soziologie des Rechts, 1947, 1964 (2. ed.), 1970 (3. ${ }^{\mathrm{a}}$ ed.), pág. 58: «Norm im eigentlichen Sinn oder subsistente Norm».

${ }^{8}$ Cfr. A. G. Conte, Aspekte der Semantik der deontischen Sprache, 1977; A. G. Conte, Aspetti della semantica del linguaggio deontico, 1977; A. G. Conte, Nove studi sul linguaggio normativo, 1985. 
Un deklarativer Normsatz parece ser simultáneamente rhético y thético:

(i) rhético, en tanto que constatación (rhética) de validez deóntica.

(ii) thético, en tanto que constitución (thética) de validez deóntica.

\subsection{Resolución de la paradoja}

2.3.1. En realidad, en el concepto de deklarativer Normsatz no hay ninguna paradoja. La apariencia paradójica del concepto de deklarativer Normsatz se disipa desde el momento en que se percibe la distinción entre validez deóntica thética y validez deóntica athética, distinción que revela así su propia fecundidad heurística.

2.3.2. Es cierto que un deklarativer Normsatz es tanto constatación (Feststellung) como constitución (Festsetzung) de validez deóntica.

Pero es falso que, en un deklarativer Normsatz, el objeto de constatación y de constitución (de athética Feststellung y de thética Festsetzung) sea una única y misma validez deóntica.

Un deklarativer Normsatz es:

(i) constatación de validez deóntica athética,

(ii) constitución de validez deóntica thética.

\section{Enunciados deónticos, enunciados adeónticos sobre la validez, lógica deóntica}

\subsection{Enunciado deóntico declarativo vs. enunciado adeóntico sobre la validez}

3.1.1. Una breve aclaración sobre lo que los enunciados deónticos declarativos ("deklarative Normsätze») de Theodor Geiger no son.

3.1.1.1. Contrariamente a lo que el adjetivo «deklarative» sugiere, los deklarative Normsätze geigerianos son (no: descriptivos de validez, sino) constitutivos de validez. (Al sintagma «constitutivo de validez» recurrí ya en Amedeo G. Conte, Studio per una teoria della validità, 1970).

La declaratividad de Geiger no es descriptividad: los deklarative Normsatze no son enunciados (enunciados deónticos, o enunciados 
adeónticos) descriptivos sobre la validez (sobre ninguna de las seis especies de validez deóntica representadas mediante los vértices del octaedro deóntico en Amedeo G. Conte, Minima deontica, 1988).

3.1.1.2. Así, el concepto (elaborado por Theodor Geiger) de deklarativer Normsatz no coincide ni con el concepto (elaborado por Hans Kelsen) de Sollsatz ni con el concepto (elaborado por Amedeo G. Conte en Studio per una teoria della validità, 1970) de enunciado deóntico descriptivo ni con el concepto (elaborado por Ingemar Hedenius, Om rätt och moral, 1941) de enunciado jurídico inauténtico (en sueco, oäkta rättssats).

\subsection{Enunciados adeónticos sobre la validez deóntica}

3.2.1. Esta breve aclaración sobre lo que los enunciados deónticos declarativos geigerianos («deklarative Normsatze») no son (no son enunciados descriptivos sobre la validez) suscita forzosamente una cuestión.

¿Cuál es el estatuto semiótico de los enunciados descriptivos sobre la validez (enunciados a cuyo conjunto los enunciados deónticos declarativos no pertenecen)?

3.2.2. Una respuesta a la cuestión del estatuto semiótico de los enunciados descriptivos sobre la validez la da un ensayo de enero de 1963: Amedeo G. Conte, La negazione di norme. Conforme a este ensayo, un enunciado descriptivo sobre la validez es un enunciado no deóntico, un enunciado adeóntico.

Un enunciado descriptivo sobre la validez (así argumentaba yo entonces, contaminando dos idiomas y combinando libremente términos alemanes con términos italianos) es un «Satz sul Sollen», y no un «Satz: di Sollen».

En tanto que enunciado sobre el Sollen, no de Sollen, un enunciado descriptivo sobre la validez es un enunciado «no modal», un enunciado amodal (enunciato amodale, amodaler Satz, amodal sentence, énoncé amodal). Un enunciado descriptivo sobre la validez es (empleo un neologismo mío de 1986: «adeontico») un enunciado adeóntico (enunciato adeontico, adeontischer Satz, adeontic sentence, énoncé adéontique).

${ }^{9}$ De mi distinción entre enunciados deónticos y enunciados adeónticos sobre la validez se distingue la distinción (también formulada en 1963) entre interpretación modal («modal interpretation») e interpretación factual ("factual interpretation») de los enunciados deónticos («norm sentences»). Tal distinción se halla en un filósofo 
3.3. Lógica deóntica, lógica de enunciados deónticos, lógica de enunciados adeónticos sobre la validez.

La tesis (que formulé en enero de 1963) de la adeonticidad de los enunciados descriptivos sobre la validez produce consecuencias inmediatas para la deóntica y para la metadeóntica. Estas consecuencias han sido destacadas tanto por Giampaolo M. Azzoni como por Paolo di Lucia.

Si los enunciados descriptivos sobre la validez son adeónticos, entonces se debe rechazar que la lógica de estos enunciados adeónticos sea una lógica que sea lógica de enunciados deónticos.

Por consiguiente, una lógica deóntica que se configure como lógica de enunciados deónticos no es (precisamente por ser construida como lógica de enunciados deónticos) lógica de enunciados descriptivos adeónticos sobre la validez.

(Traducción de Carlos Alarcón Cabrera)

finlandés de lengua sueca: Erik Stenius, The Principles of a Logic of Normative Systems, 1963.

La analogía entre las dos distinciones es sólo aparente. En efecto, Stenius no distingue dos especies de enunciados, sino dos especies de interpretaciones de enunciados.

En otras palabras: en Stenius, «modal» y «factual» se predican de interpretaciones de enunciados, no de enunciados.

Con otras palabras diferentes: en Stenius, son modales (o, respectivamente, amodales) no los enunciados, sino las interpretaciones de enunciados.

En el origen de la tesis de Stenius y de una tesis análoga de Georg Henrik von Wright que también se remonta a 1963 (G. H. Von Wright, Norm and Action, 1963) se encuentra, según el propio Stenius (y también según Von Wright), el libro del filósofo sueco Ingemar Hedenius, Om rätt och moral, 1941 (äkta rättssats vs. oäkta rättssats, enunciado jurídico auténtico vs. enunciado jurídico inauténtico).

Un curiosum: en 1963, tanto Stenius como von Wright y Conte (por este orden) habían estado ya, por diferentes razones (y a diferentes niveles), en relación con la obra de Ludwig Wittgenstein. 


\section{REFERENCIAS BIBLIOGRÁFICAS}

T. W. Adorno, Philosophische Terminologie, Frankfurt am Main. Suhrkamp. 1974.

G. P. M. Azzoni, Il concetto di condizione nella tipologia delle regole, Padova, CEDAM, 1988.

R. Brown, Rules and Laws in Sociology, London, Routledge, 1973.

A. G. Conte, La negazione di norme, Pavia, enero 1963. [Manuscrito].

A. G. Conte, Studio per una teoria della validità, en «Rivista intemazionale di filosofia del diritto», 47 (1970), págs. 331-354.

A. G. Conte, Codici deontici, en Intorno al «codice». Atti del terzo convegno della Associazione italiana di studi semiotici AISS (Pavia, 1975), Firenze, La Nuova Italia, 1976, pp. 13-25. Reedición en: A. G. Conte, Nove studi sul linguaggio normativo, Torino, Giappichelli, 1985, págs. 131-145.

A. G. Conte, Aspekte der Semantik der deontischen Sprache, en A. G. Conte/Risto Hilpinen/Georg Henrik von Wright (eds.), Deontische Logik und Semantik, Wiesbaden, Athenaion, 1977, págs. 59-73.

A. G. Conte, Aspetti della semantica del linguaggio deontico, en Giuliano di Bernardo (ed.), Logica deontica e semantica, Bologna, Il Mulino, 1977, págs. 147-175.

A. G. Conte, Nove studi sul linguaggio normativo, Torino, Giappichelli, 1985.

A. G. Conte, Minima deontica, en «Rivista internazionale di filosofia del diritto», 65 (1988), págs. 425-475.

P. Di Lucia, Momenti della deontica di von Wright, en «Rivista trimestrale di diritto e procedura civile», 44 (1990).

C. Donati, Note sul normativismo, Milano, Giuffrè, 1975.

C. Donati (ed.), Dizionario critico del diritto, Milano, Savelli, 1980.

A. Febbrajo, Materiali sulla sociologia del diritto di Theodor Geiger, Pavia, GJES, 1979.

J.-L. Gardies, Norme et nature, en Werner Krawietz (ed.), Theorie der Normen, Berlin, Duncker und Humblot, 1984, págs. 105-117.

J.-L. Gardies, L'erreur de Hume, Paris, Presses Universitaires de France, 1987.

T. Geiger, Vorstudien zu einer Soziologie des Rechts, Kobenhavn, E. Munksgaard, 1947; Neuwied, Luchterhand, 1964 (2a. ed.); Neuwied, Luchterhand, 1970 (3a ed.). Traducción española de Arturo Camacho, Guillermo Hirata y Ricardo Orozco, Estudios de sociología del derecho, México D.F., Fondo de Cultura Económica, 1983.

F. A. von Hayek, Law, Legislation and Liberty, 1982, London, Routledge.

I. Hedenius, Om rätt och moral, Stockholm, Tidens förlag, 1941; Stockholm, Wahlstrom och Windstrand, 1963 (2. ${ }^{\mathrm{a}}$ ed.), 1965 (3. ${ }^{\mathrm{a}}$ ed.).

G. Kalinowsky, Ontique et déontique, en «Rivista internazionale di filosofia del diritto», 66 (1989), págs. 437-449.

A. Kemmerling, Regel und Geltung im Lichte der Analyse Wittgensteins, en «Rechtstheorie», 6 (1975), págs. 104-131.

P. Kiparksy/C. Kiparki, Fact, en: Manfred Bierwisch/Karl Erich Heidolth (eds.), Progress in Linguistics, The Hague, Mouton, 1970, págs. 143-173. 
L. Lombardi Vallauri, Saggio sul diritto giurisprudenziale, Milano, Giuffrè, 1967.

E. Stenius, The Principles of a Logic of Normative Systems, en «Acta philosophica fennica», 16 (1963), págs. 247-260.

E. Stenius, Ross Paradox and Well-Formed Codices, en «Theoria», 48 (1982), págs. 49-77.

G. Tarello, Teorie e ideologie nel diritto sindacale, Milano, Comunità, 1967.

M. E. Viora, Consolidazioni e codificazioni, Bologna, Zanichelli, 1932; Torino, Giappichelli, 1967 (3. ${ }^{\mathrm{a}}$ ed.)

G. H. von Wright, Norm and Action, London, Routledge, 1963. 